\title{
Protective effects of resveratrol on autologous nucleus pulposus model of radiculopathy
}

\author{
BIN LIN, HUI YU, YONGZHI HE, YANG XU, WENBIN ZHANG, CHENGWU LU and QINGFANG AO \\ Department of Orthopaedics, The 175th Hospital of PLA, Southeast Hospital of Xiamen University, \\ Zhangzhou, Fujian 363000, P.R. China
}

Received September 26, 2014; Accepted October 8, 2016

DOI: $10.3892 /$ etm.2016.3878

\begin{abstract}
Nucleus pulposus (NP) has been suggested to trigger an autoimmune response if exposed to the immune system, which plays a key role in neuropathic pain. Therefore, appropriate suppression of inflammation is a key factor for treating the radiculopathy caused by intervertebral disk (IVD) degeneration. Resveratrol, a key component of red wine, has been suggested to exhibit anti-inflammatory properties in vitro and in vivo. However, the effects of resveratrol on NP-mediated pain in vivo have not been studied. The aim of the present study was to investigate whether resveratrol may be useful in treating NP-mediated pain in an autologous NP model of radiculopathy. A total of 36 adult male Sprague-Dawley rats were allocated randomly into sham (group I), saline-treated (group II) and resveratrol-treated (group III) groups. Animal behavior in response to non-noxious mechanical stimulation with von Frey filaments was compared at days 0 (baseline), 3, 7,14 and 21 following surgery. The expression of proinflammatory cytokines such as tumor necrosis factor $\alpha(\mathrm{TNF}-\alpha)$ and interleukin-1 (IL-1) were assessed at days 7 and 14. The data showed that resveratrol exhibited an anti-inflammatory effect on the expression of proinflammatory cytokines. Compared with group II, the expression of TNF- $\alpha$ and IL-1 was significantly decreased at each time point in group III. In addition, resveratrol significantly reduced pain behavior triggered by the application of NP tissue on the dorsal root ganglion for up to 14 days. These data suggest that resveratrol has potential for the treatment of NP-mediated pain, indicating a potential clinical application.
\end{abstract}

Correspondence to: Dr Bin Lin, Department of Orthopaedics, The 175th Hospital of PLA, Southeast Hospital of Xiamen University, 269 Zhanghua Road, Zhangzhou, Fujian 363000, P.R. China E-mail: linbin813@163.com

Key words: resveratrol, anti-inflammatory, nucleus pulposus-mediated back pain, radiculopathy

\section{Introduction}

Nucleus pulposus (NP) has been considered to be excluded from the development of immunological tolerance as this part of the intervertebral disc (IVD) normally has no access to systemic circulation (1). Therefore, NP has been suggested to trigger an autoimmune response if exposed to the immune system, which has long been known to be a potent inducer of pain (2). Historically, mechanical pressure was considered to be the sole pathophysiologic mechanism for inducing sciatica, when Mixter and Barr (3) linked the onset of pain to the mechanical compression of the nerve root. However, biochemical factors derived from the NP, such as TNF- $\alpha$ and IL-1, have subsequently been found to be an additional pathophysiologic mechanism for inducing pain (4). The current treatment options of NP-mediated pain are not ideal, as they target symptomatic relief but do not interfere with the biological mechanisms underlying pain development (5). In clinical practice, reducing the inflammation can notably ameliorate the clinical outcomes of some autoimmune diseases $(6,7)$, suggesting that the reduction of proinflammatory cytokine production may serve as an efficient therapeutic target for NP-mediated pain. Hence, an ideal anti-inflammatory should be selected with the ability to interfere with the biological mechanisms of NP-mediated pain, thus limiting further degeneration of the IVD.

There is a growing interest in natural bioactive compounds that may have a pronounced anti-inflammatory effects, similar to those of corticosteroids (8). Resveratrol (3,5,4'-trihydroxy-trans-stilbene) is a polyphenolic, antifungal natural phytoalexin found in various food products, with particularly high levels in grape skin and red wine. Resveratrol has been shown to have anti-inflammatory, antioxidant, antitumorigenic and immunomodulatory properties (9-11). Recently, resveratrol has been reported to exert direct cardiovascular protective effects by improving myocardial perfusion, reducing oxidant stress and inhibiting platelet aggregation (12-14). However, it is not known if resveratrol exerts similar protective effects on the NP-mediated pain. On the basis of these findings, we hypothesized that resveratrol may exerts similar protective effects on degenerating IVDs.

Furthermore, a variety of inflammatory mediators have been implicated in IVD degeneration, including nitric oxide (NO), interleukin-1 (IL-1), matrix metalloproteinases (MMP), prostaglandin E2 (PGE2), tumor necrosis factor $\alpha(\mathrm{TNF}-\alpha)$ and 
various other cytokines $(15,16)$. There is some direct evidence that the key mediators of these changes may be TNF- $\alpha$ and IL-1 (17-19), which are expressed at higher levels in herniated discs compared with degeneration matched controls $(20,21)$. In addition, systemic immunomodulatory treatment targeting of TNF- $\alpha$ activity attenuates these effects in animal models (22-25).

On the basis of the obvious requirement for more defined, biological treatment options for NP-mediated pain, we hypothesized that resveratrol may react with proinflammatory cytokine to relieve the NP-mediated pain. Hence, the purpose of this study was to investigate the protective effects of resveratrol on NP cells with regard to proinflammatory cytokines.

\section{Materials and methods}

Ethical approval. All experimental procedures were performed in accordance with protocols approved by the Governmental Animal Care Committee of the Medical College of Xiamen University (Zhangzhou, China) and conformed to the National Institute of Health guidelines on the ethical use of animals.

Model of radiculopathy. Prior surgery, the animals were anesthetized by intraperitoneal injection of $400 \mathrm{mg} / \mathrm{kg}$ chloral hydrate (Beyotime Institute of Biotechnology, Haimen, China). During surgery, the rats were placed in a prone position on a warming pad to maintain a body temperature of $37.0 \pm 0.5^{\circ} \mathrm{C}$. After surgery, the animals were returned to individual cages with sufficient water and food and treated with an intramuscular injection of penicillin (The 175th Hospital of PLA, Zhangzhou, China) at a dose of 200,000 U/day, for three days. After $72 \mathrm{~h}$, rats were sacrificed by anesthetic overdose, with the dose depending on the degree of tolerance to chloral hydrate.

Adult female Sprague-Dawley rats $(n=36$; weight, 240-260 g) were randomly assigned into three groups $(n=12$ per group): Sham injury (group I), saline-treated (group II) and resveratrol-treated (group III). Resveratrol was obtained from the Xi'an Xiaocao Botanical Institute of Biotechnology (Xi'an, China). The rats were obtained from the Experimental Animal Center of Xiamen University.

The Autologous Nucleus Pulposus Model of Radiculopathy was established as described in a previous study (26). Briefly, after the skin preparation of the lower back, an incision was made at the L4-S1 level, fascia and multifidus muscle were resected, exposing the L4 and L5 vertebral laminae, and the left L5 nerve root and dorsal root ganglion (DRG) were exposed by L5-L6 hemilaminectomy on the left side. Care was taken to avoid trauma to the tissue. After identifying the annuli fibrosi, autologous NP harvested from the tail was applied to the DRG in the saline treatment group and the resveratrol treatment group. In the Sham group, the left L5 nerve root and DRG were exposed by L5-L6 facetectomy on the left side without no other procedures were performed.

Resveratrol was administered to the rats in group III at a dose of $0.1 \mathrm{ml} 50 \mu \mathrm{M}$ solution in saline, while in group II $0.1 \mathrm{ml}$ saline was injected into the underlayer of the epineurium just distal to the NP prior to closing the incisions. Group I rats received no treatment throughout the experiment.

Behavioral testing. The behavioral testing was evaluated by the paw withdrawal threshold of the left hind limb. At days 0 (base- line), 3, 7, 14 and 21, all animals underwent behavioral testing by evaluating the left hind paw withdrawal response to von Frey hair stimulation of the plantar surface of the footpad. Briefly, von Frey filaments (27) with a calibrated mechanical stimuli (Stoelting Co., Wood Dale, IL, USA) between 1 and $29 \mathrm{~g}$ were sequentially applied twice to the paw surface. The determined withdrawal force was verified with a negative test of next lower filament as well as by confirming the initial response after a time lag of $5 \mathrm{~min}$. Lower withdrawal thresholds was considered to be a sign of mechanical hypersensitivity, which is correlated to pain behavior in this animal model.

Hematoxylin and eosin $(H E)$ staining for detecting pathological changes. For HE staining, $5-\mu \mathrm{m}$ transverse sections $(8 \mu \mathrm{m})$ of tissue at 7 and 14 days post surgery in each group were deparaffinized and put into fresh xylene for $15 \mathrm{~min}$ twice. Sections were rehydrated in $100 \%$ alcohol for 5 min twice, then $95 \%$ alcohol and $70 \%$ alcohol once for $3 \mathrm{~min}$. Subsequently, sections were washed briefly in $\mathrm{ddH}_{2} \mathrm{O}$, stained in Harris hematoxylin (Beyotime Institute of Biotechnology) solution for $5 \mathrm{~min}$. Sections were then washed in running tap water for $8 \mathrm{~min}$ and differentiated in $1 \%$ acid alcohol for $30 \mathrm{sec}$, blued in $0.2 \%$ ammonia water for $30 \mathrm{sec}$. The sections were washed in running tap water for $5 \mathrm{~min}$ and rinsed in $95 \%$ alcohol $\sim 15$ times. Sections were stained in Eosin-Phloxine (Beyotime Institute of Biotechnology) solution for $1 \mathrm{~min}$ then dehydrated through 95 and $100 \%$ alcohol $(5 \mathrm{~min}$ each) and cleared in two changes of xylene (5 min each). Finally, the sections were mounted with mounting medium (Beyotime Institute of Biotechnology). Images were captured using a FV 300 confocal microscope (Olympus Corporation, Tokyo, Japan).

Immunohistochemical staining. For immunohistochemical staining, each specimen was embedded in paraffin and six serial sections (three for TNF- $\alpha$ and three for IL-1) were cut using a microtome. The immunohistochemical study of TNF- $\alpha$ and IL-1 was performed using an avidin-biotin peroxidase complex technique and Histostain SP kit (Maixin-Bio, Inc., Fuzhou, China) according to the manufacturer's instructions. Mouse anti-TNF- $\alpha$ (1:1,000; cat. no. 23456-1-AP; Endogen, Inc., Rockford, IL, USA) and anti-IL-1 polyclonal antibody (1:100; cat. no. abM50001-5G3-PU; Santa Cruz Biotechnology, Inc., Santa Cruz, CA, USA) were used for this study. Two pathologists who were unaware of the experimental data were responsible for counting the TNF- $\alpha$ - and IL-1-positive cells in 10 high-power fields (magnification, $x 400$ ) in each specimen. Finally, the total number of IL- 1 and TNF- $\alpha$-positive cells per section were counted. Images were captured using the FV 300 confocal microscope.

Statistical analysis. Statistical analysis was performed using SPSS version 13.0 for Windows (SPSS, Inc., Chicago, IL, USA). Data are presented as the mean \pm standard deviation. The Mann-Whitney U-test were used for statistical analyses. $\mathrm{P}<0.05$ was considered to indicate a statistically significant difference.

\section{Results}

Surgery outcome. In all rats, the rectal temperature was maintained at $37 \pm 0.5^{\circ} \mathrm{C}$ during surgery. The mean body weight of sham operated rats was $371.25 \pm 6.81 \mathrm{~g}$ (range, 362-385 g; $\mathrm{n}=12$ ). 


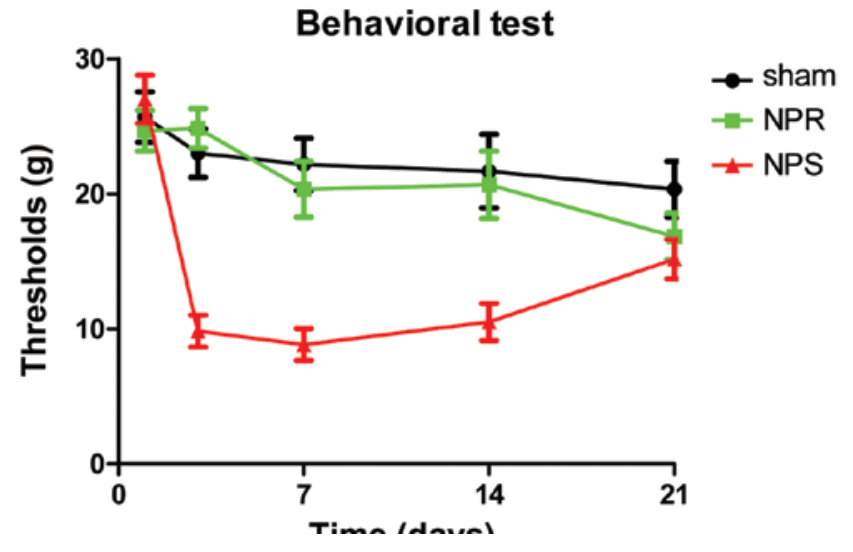

Figure 1. Resveratrol reduces pain behavior in vivo. Compared with group I, group II reduced mechanical sensitivity thresholds primarily up to day 14 . In group III, thresholds were significantly higher than in group II at days 3,7 and $14(\mathrm{P}<0.01)$ and similar to group I (indicative of pain reduction by resveratrol treatment). Data were obtained by von Frey filament testing and are presented as the mean \pm standard deviation $(n=6)$. NPR, resveratrol-treated group; NPS, saline-treated group.

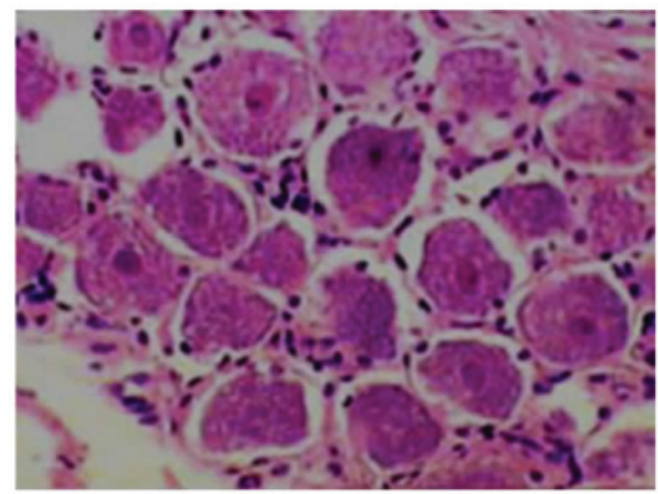

Figure 2. Day 7 post surgery in group I showed a normal appearance as expected (stain, hematoxylin \& eosin; magnification, x200).

The mean body weights of the control and U0126 groups were $377.67 \pm 7.1 \mathrm{~g}$ (range, $367-388 \mathrm{~g} ; \mathrm{n}=12$ ) and $371.75 \pm 10.00 \mathrm{~g}$ (range, 354-389; $n=12$ ), respectively. All rats underwent the surgical procedure without complications. No significant differences in physiological parameters were detected between the groups.

Behavioral test. To evaluate the extent of motor function recovery, non-noxious mechanical stimulation with von Frey filaments was employed. Animal behavior in response to the stimulation was assessed for the three groups at different time points after surgery. In group I, which (as expected) showed stable mechanical withdrawals that were overall close to the preoperative baseline over the entire course of the experiment, indicating that the surgical intervention alone did not cause any change in pain behavior. Groups II and III were compared with group I for all time points. Results indicate that in group II, the mechanical withdrawal thresholds were significantly decreased for each time point compared with the baseline values of the sham group up to day 14 , indicating that pain was evoked by application of NP tissue to the DRG. While in group III that was treated with resveratrol, animal

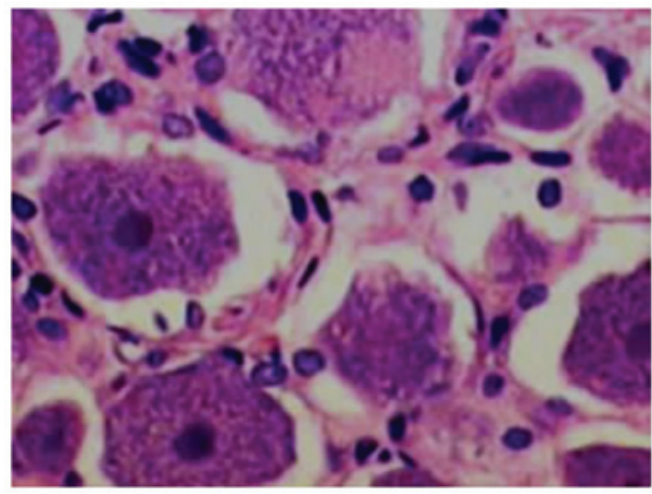

Figure 3. Day 7 post surgery in group II showing cellular edema of dorsal root ganglion cells, irregular structure of cytoplasm, nissl body population decreased and focal hyperaemia, which indicated the inflammatory response (stain, hematoxylin \& eosin; magnification, x400).

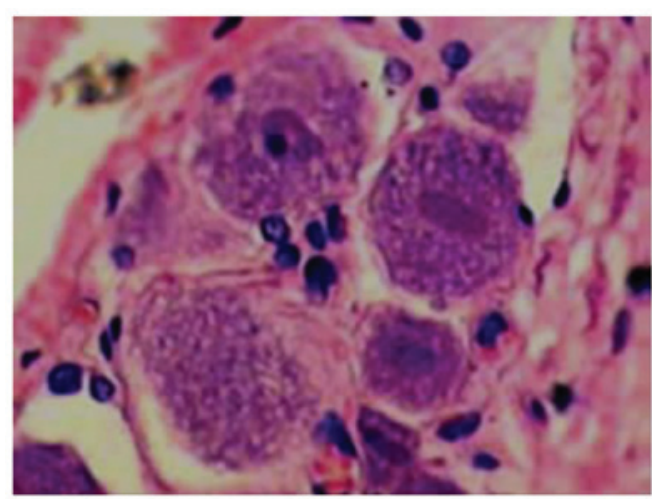

Figure 4. Day 7 post surgery, the resveratrol-treated rats (group III) showed improved cell structure, with decreased edema and focal hyperaemia compared with group II (stain, hematoxylin \& eosin; magnification, x400).

behavior was very similar to group I. Thresholds in group III were significantly higher compared with group II on days 3 , 7 and $14(\mathrm{P}<0.01)$. However, at day 21 , there was no significant difference anymore between the thresholds of groups II and III (Fig. 1).

Visual study. Following surgery, there was no obvious tissue oedema in group I, indicating that the surgical intervention alone did not cause any change in the DRG and IVD. While in group II and III, obvious tissue oedema was observed in at 7 days post surgery and was visibly attenuated at 14 days post surgery.

$H \& E$ staining. In group I, neurons had a normal appearance, as expected (Fig. 2). In group II at 7 and 14 days post surgery, morphological detection indicated increased inflammatory response in the neurons: Cellular edema of DRG cells, irregular structure of cytoplasm, nissl body population decreased and focal hyperaemia (Fig. 3). In group III, cell structure was improved, with decreased edema and focal hyperaemia compared with group II (Fig. 4). Mild inflammatory response was detected in the focal zone, but not as obvious or serious as in the group II. In summary, the DRG pathological changes that occurred after surgery were significantly attenuated by resveratrol at 7 and 14 days post surgery. 
Table I. TNF- $\alpha$-positive cells per section.

\begin{tabular}{lcc}
\hline & \multicolumn{2}{c}{ Post-surgery (days) } \\
\cline { 2 - 3 } Group & 7 & 14 \\
\hline I & $3.00 \pm 1.41$ & $2.67 \pm 1.03$ \\
II & $24.00 \pm 2.61^{\mathrm{a}}$ & $20.83 \pm 2.32^{\mathrm{a}}$ \\
III & $22.83 \pm 2.48^{\mathrm{a}, \mathrm{b}}$ & $9.17 \pm 2.04^{\mathrm{a}, \mathrm{b}}$ \\
\hline
\end{tabular}

${ }^{\mathrm{a}} \mathrm{P}<0.01$ vs. group I; ${ }^{\mathrm{b}} \mathrm{P}<0.05$ vs. group II. Data presented as the mean \pm standard deviation. TNF- $\alpha$, tumor necrosis factor- $\alpha$; group I, sham group; group II, saline treatment group; group III, resveratrol treatment group. TNF- $\alpha$, tumor necrosis factor- $\alpha$.

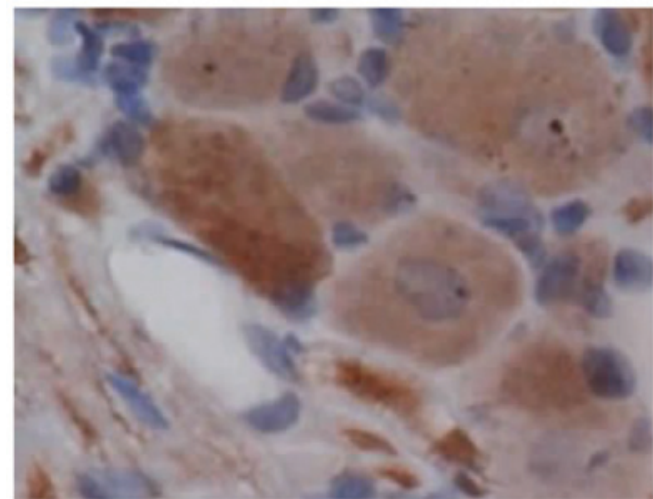

Figure 5. Day 7 post surgery, the expression of tumor necrosis factor- $\alpha$ was detected at a low level by immunofluorescence in the sham rats (group I) (magnification, $\mathrm{x} 400$ ).

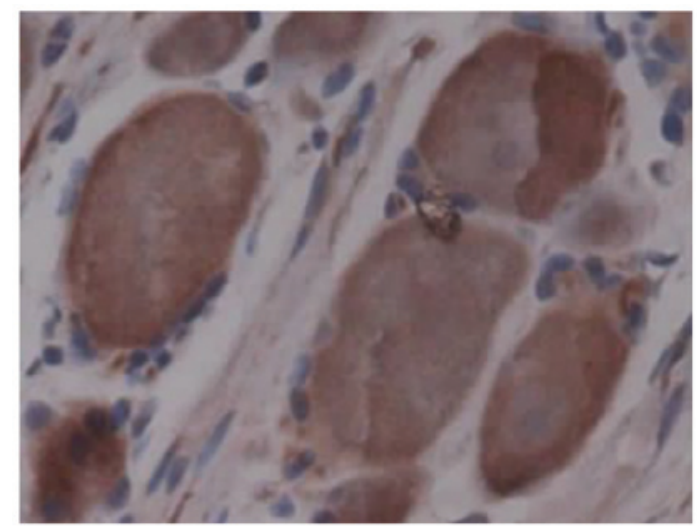

Figure 6. Day 7 post surgery, the expression of tumor necrosis factor- $\alpha$ was detected at a high level in the saline-treated rats (group II) (magnification, x400).

Effects of resveratrol on the protein expression levels of TNF- $\alpha$ and $I L-1$. Increased constitutive expression of TNF- $\alpha$ and IL-1 was observed in groups II and III (Tables I and II). However, TNF- $\alpha$ and IL-1 immunohistochemical analysis of tissue sections showed significant differences between groups II and III. Compared with group II, resveratrol markedly inhibited the expression of TNF- $\alpha$ and IL-1 was observed at 7 and 14 days post surgery in group III. This result indicates that the anti-inflammatory effect of the highest concentration of
Table II. IL-1-positive cells per section.

\begin{tabular}{lcc}
\hline & \multicolumn{2}{c}{ Post-surgery (days) } \\
\cline { 2 - 3 } Group & \multicolumn{1}{c}{14} \\
\hline I & $2.5 \pm 1.05$ & $1.83 \pm 1.17$ \\
II & $23.67 \pm 1.75^{\text {a }}$ & $20.50 \pm 2.07^{\mathrm{a}}$ \\
III & $23.67 \pm 4.18^{\mathrm{a}}$ & $9.67 \pm 3.08^{\mathrm{a}, \mathrm{b}}$
\end{tabular}

${ }^{\mathrm{a}} \mathrm{P}<0.01$ vs. group I; ${ }^{\mathrm{b}} \mathrm{P}<0.05$ vs. group II. Data presented as the mean \pm standard deviation. IL-1, interleukin 1; group I, sham group; group II, saline treatment group; group III, resveratrol treatment group. IL-1, interleukin-1.

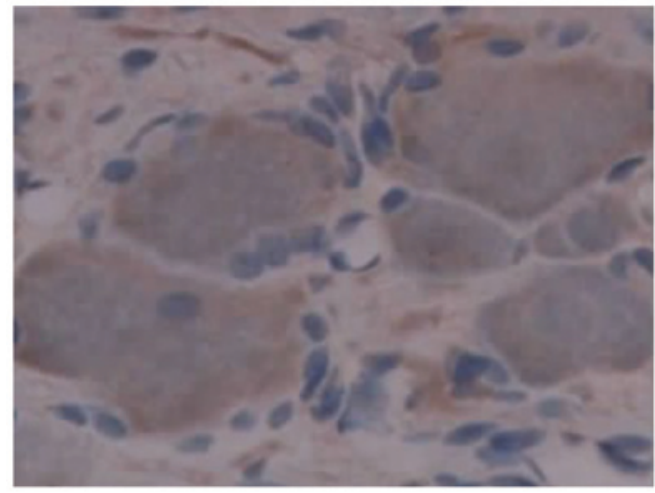

Figure 7. Day 7 post surgery expression of tumor necrosis factor (TNF)- $\alpha$ was detected by immunofluorescence in resveratrol-treated rats (group III). Compared with the saline treatment group, resveratrol inhibited the expression of TNF- $\alpha$ (magnification, $\mathrm{x} 400)$.

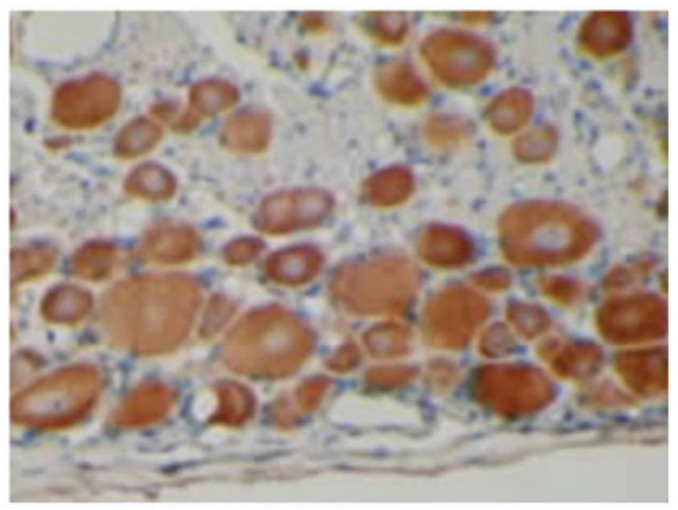

Figure 8 . Day 7 post surgery, the expression of interleukin- 1 was significantly increased in the saline-treated rats (group II) compared with the sham rats (group I) (magnification, x200).

resveratrol could be confirmed on the protein level for TNF- $\alpha$ and IL-1 (Figs. 5-9).

\section{Discussion}

Lower back pain remains a major public health concern worldwide. Lower back pain is among the most common reasons for visiting a doctor and is the most common cause of disability in patients $<45$ years old (28). Amongst a variety of etiologies, 


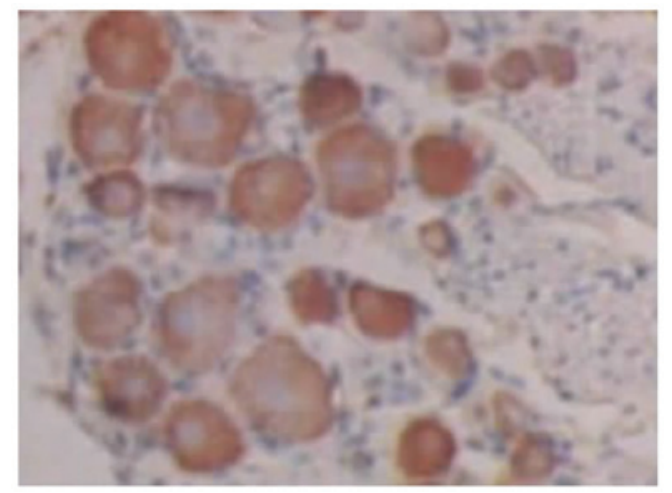

Figure 9. Day 7 post surgery, the expression of interleukin (IL)-1 was detected by immunofluorescence in the resveratrol-treated rats (group III). Compared with the saline treatment group, resveratrol inhibited the expression of IL-1 (magnification, x200).

degenerative disc disease (leading to so-called discogenic back pain) that correlated with increased levels of proinflammatory cytokines has been postulated to be a crucial cause of lower back pain $(21,29,30)$. Current treatments for lower back pain remain contested, indicating that current treatment options are not ideal, as current therapeutic strategies do affect the underlying biological mechanisms of pain development (5). For those reasons, injectable anti-inflammatory substances that specifically target the metabolism of IVD cells may serve as novel and useful minimally-invasive treatment options $(31,32)$.

TNF- $\alpha$ has been reported to produce radicular pain caused by lumbar disc herniation in adult patients, as mentioned earlier (22-24). Recently, cytokines such as IL-1, IL-6, and TNF- $\alpha$ have been associated with lower back and radicular pain $(7,33)$. The cytokines IL-1 and TNF- $\alpha$ are overexpressed in degenerated IVD, which has led to them being implicated in the matrix degradation that characterizes disk degeneration (34-36). Furthermore, it has been reported that a single intravenous infusion of the TNF- $\alpha$ inhibitor infliximab was effective in treating sciatic pain caused by lumbar disc herniation in clinical practice (20). Cohen et al (37) reported a preclinical safety study of transforaminal epidural etanercept (another TNF- $\alpha$ inhibitor) for the treatment of sciatica caused by disc herniation in 24 patients. The results indicated that the effectiveness was dependent on the dose of etanercept. Among these, proinflammatory cytokines such as TNF- $\alpha$ and IL-1 have been the focus of a number of studies investigating the pathogenesis of intervertebral disc degeneration, herniation and sciatic pain (22-24).

There is growing evidence over the past decade that resveratrol may impact multiple biological systems, particularly the immune system (9-12). Recently, resveratrol has gained considerable attention due to its anticancer (38), cardiovascular protective (39) and anti-inflammatory effects (40). A previous study has shown that resveratrol can effectively reduce mRNA levels of major proinflammatory cytokines (IL-6 and IL-8), TLR2, and matrix degrading enzymes (MMP1, MMP3 and MMP13) (41), which have previously been shown to be involved in disc degeneration and pain induction (42). Furthermore, recent studies demonstrate that resveratrol can effectively prevent and treat experimental inflammatory diseases by suppressing the production of inflammatory cytokines in various types of cells (43-45). Notably, in a study performed by Li et al (46), resveratrol has additionally been shown to increase proteoglycan synthesis and to reduce TNF- $\alpha$ - and IL-1-induced proteoglycan loss in bovine IVD cells, therefore providing further evidence that resveratrol may be an innovative treatment for NP-mediated back and leg pain $(16,41,46)$.

The present study investigated the effect of resveratrol on autologous NP model of radiculopathy as an in vivo model. This animal model of radiculopathy involved placing autologous tail NP tissue onto an exposed lumbar DRG in treated animals. The advantage of this model is that using tail NP ensures that the lumbar disc is neither injured by annular puncture nor weakened by NP evacuation. This aids evaluation of inflammatory and immune changes at the DRG specific to the presence of autologous NP, particularly when annular incision alone can cause radiculopathy in animal models (47-49).

As shown in the present study, resveratrol suppresses the expression of TNF- $\alpha$ and the TNF- $\alpha$-induced production of IL-1. The present results also suggest that resveratrol may have potential as a treatment for NP-mediated back and leg pain. Resveratrol treatment was able to prevent the pain-related behavior to a certain degree for 14 days in rats. This may be due to resveratrol reducing or inhibiting the expression cytokines that are released from the NP tissue in vivo, similar to the mechanism observed in previous in vitro cell culture studies $(41,46)$.

In conclusion, this study has underlined the potential of resveratrol for attenuating NP-mediated pain. Since resveratrol is clinically widely used, its beneficial effects in preventing and treating IVD can be transplanted to the bedside. Resveratrol may provide a new therapeutic approach in treatment of IVD.

\section{References}

1. Geiss A, Larsson K, Rydevik B, Takahashi I and Olmarker K: Autoimmune properties of nucleus pulposus: An experimental study in pigs. Spine (Phila Pa 1976) 32: 168-173, 2007.

2. Suzuki M, Inoue G, Gemba T, Watanabe T, Ito T, Koshi T, Yamauchi K, Yamashita M, Orita S, Eguchi Y, et al: Nuclear factor-kappaB decoy suppresses nerve injury and improves mechanical allodynia and thermal hyperalgesia in a rat lumbar disc herniation model. Eur Spine J 18: 1001-1007, 2009.

3. Mixter WJ and Barr J: Rupture of the intervertebral disc with involvement of the spinal canal. N Engl J Med 211: 210-215, 1934

4. Olmarker K and Larsson K: Tumor necrosis factor alpha and nucleus-pulposus-induced nerve root injury. Spine (Phila Pa 1976) 23: 2538-2544, 1998.

5. Sharifi S, Bulstra SK, Grijpma DW and Kuijer R: Treatment of the degenerated intervertebral disc; closure, repair and regeneration of the annulus fibrosus. J Tissue Eng Regen Med 9: 1120-1132, 2015.

6. Ancuţa C, Ancuţa E, Miu S, Iordache C, Belibou C and Chirieac R: Adalimumab therapy in patients with active rheumatoid arthritis. Rev Med Chir Soc Med Nat Iasi 113: 710-715, 2009.

7. Yount S, Sorensen MV, Cella D, Sengupta N, Grober J and Chartash EK: Adalimumab plus methotrexate or standard therapy is more effective than methotrexate or standard therapies alone in the treatment of fatigue in patients with active, inadequately treated rheumatoid arthritis. Clin Exp Rheumatol 25: 838-846, 2007.

8. Fontana G, See E and Pandit A: Current trends in biologics delivery to restore intervertebral disc anabolism. Adv Drug Deliv Rev 84: 146-158, 2015.

9. Harikumar KB and Aggarwal BB: Resveratrol: A multitargeted agent for age-associated chronic diseases. Cell Cycle 7: 1020-1035, 2008. 
10. Soleas GJ, Diamandis EP and Goldberg DM: The world of resveratrol. Adv Exp Med Biol 492: 159-182, 2001.

11. Burns J, Yokota T, Ashihara H, Lean ME and Crozier A: Plant foods and herbal sources of resveratrol. J Agric Food Chem 50: 3337-3340, 2002.

12. Schmitt CA, Heiss EH and Dirsch VM: Effect of resveratrol on endothelial cell function: Molecular mechanisms. Biofactors 36: 342-349, 2010.

13. Robich MP, Osipov RM, Nezafat R, Feng J, Clements RT, Bianchi C, Boodhwani M, Coady MA, Laham RJ and Sellke FW: Resveratrol improves myocardial perfusion in a swine model of hypercholesterolemia and chronic myocardial ischemia. Circulation 122 (Suppl 11): S142-S149, 2010.

14. Crescente M, Jessen G, Momi S, Höltje HD, Gresele P, Cerletti $\mathrm{C}$ and de Gaetano $\mathrm{G}$ : Interactions of gallic acid, resveratrol, quercetin and aspirin at the platelet cyclooxygenase- 1 level. Functional and modelling studies. Thromb Haemost 102 336-346, 2009.

15. Cassinelli EH, Hall RA and Kang JD: Biochemistry of intervertebral disc degeneration and the potential for gene therapy applications. Spine J 1: 205-214, 2001.

16. Kang JD, Stefanovic-Racic M, McIntyre LA, Georgescu HI and Evans $\mathrm{CH}$ : Toward a biochemical understanding of human intervertebral disc degeneration and herniation. Contributions of nitric oxide, interleukins, prostaglandin E2, and matrix metalloproteinases. Spine (Phila Pa 1976) 22: 1065-1073, 1997.

17. Murata Y, Onda A, Rydevik B, Takahashi K and Olmarker K: Distribution and appearance of tumor necrosis factor-alpha in the dorsal root ganglion exposed to experimental disc herniation in rats. Spine (Phila Pa 1976) 29: 2235-2241, 2004.

18. Takahashi N, Kikuchi S, Shubayev VI, Campana WM and Myers RR: TNF-alpha and phosphorylation of ERK in DRG and spinal cord: Insights into mechanisms of sciatica. Spine (Phila Pa 1976) 31: 523-529, 2006.

19. Aoki Y, Rydevik B, Kikuchi S and Olmarker K: Local application of disc-related cytokines on spinal nerve roots. Spine (Phila Pa 1976) 27: 1614-1617, 2002.

20. Weiler C, Nerlich AG, Bachmeier BE and Boos N: Expression and distribution of tumor necrosis factor alpha in human lumbar intervertebral discs: A study in surgical specimen and autopsy controls. Spine (Phila Pa 1976) 30: 44-53; discussion 54,2005

21. Le Maitre CL, Hoyland JA and Freemont AJ: Catabolic cytokine expression in degenerate and herniated human intervertebral discs: IL-1beta and TNFalpha expression profile. Arthritis Res Ther 9: R77, 2007.

22. Murata Y, Olmarker K, Takahashi I, Takahashi K and Rydevik B: Effects of selective tumor necrosis factor-alpha inhibition to pain-behavioral changes caused by nucleus pulposus-induced damage to the spinal nerve in rats. Neurosci Lett 382: 148-152, 2005.

23. Murata Y, Onda A, Rydevik B, Takahashi K and Olmarker K Selective inhibition of tumor necrosis factor-alpha prevents nucleus pulposus-induced histologic changes in the dorsal root ganglion. Spine (Phila Pa 1976) 29: 2477-2484, 2004

24. Olmarker K and Rydevik B: Selective inhibition of tumor necrosis factor-alpha prevents nucleus pulposus-induced thrombus formation, intraneural edema, and reduction of nerve conduction velocity: Possible implications for future pharmacologic treatment strategies of sciatica. Spine (Phila Pa 1976) 26: 863-869, 2001

25. Onda A, Yabuki S and Kikuchi S: Effects of neutralizing antibodies to tumor necrosis factor-alpha on nucleus pulposus-induced abnormal nociresponses in rat dorsal horn neurons. Spine (Phila Pa 1976) 28: 967-972, 2003.

26. Shamji MF, Allen KD, So S, Jing L, Adams SB Jr, Schuh R, Huebner J, Kraus VB, Friedman AH, Setton LA and Richardson WJ: Gait abnormalities and inflammatory cytokines in an autologous nucleus pulposus model of radiculopathy. Spine (Phila Pa 1976) 34: 648-654, 2009.

27. Chaplan SR, Bach FW, Pogrel JW, Chung JM and Yaksh TL: Quantitative assessment of tactile allodynia in the rat paw J Neurosci Methods 53: 55-63, 1994.

28. Woodwell D: National ambulatory medical care survey: 1996 summary. Adv Data 17: 1-25, 1997.

29. Freemont AJ: The cellular pathobiology of the degenerate intervertebral disc and discogenic back pain. Rheumatology (Oxford) 48: 5-10, 2009
30. Hayashi S, Taira A, Inoue G, Koshi T, Ito T, Yamashita M, Yamauchi K, Suzuki M, Takahashi K and Ohtori S: TNF-alpha in nucleus pulposus induces sensory nerve growth: A study of the mechanism of discogenic low back pain using TNF-alpha-deficient mice. Spine (Phila Pa 1976) 33: 1542-1546, 2008.

31. An HS, Thonar EJ and Masuda K: Biological repair of intervertebral disc. Spine (Phila Pa 1976) 28 (Suppl 15): S86-S92, 2003.

32. An H, Boden SD, Kang J, Sandhu HS, Abdu W and Weinstein J: Summary statement: Emerging techniques for treatment of degenerative lumbar disc disease. Spine (Phila Pa 1976) 28 (Suppl 15): S24-S25, 2003.

33. Burke JG, Watson RW, McCormack D, Dowling FE, Walsh MG and Fitzpatrick JM: Intervertebral discs which cause low back pain secrete high levels of proinflamatory mediators. J Bone Joint Surg Br 84: 196-201, 2002.

34. Le Maitre CL, Freemont AJ and Hoyland JA: The role of interleukin-1 in the pathogenesis of human intervertebral disc degeneration. Arthritis Res Ther 7: R732-R745, 2005.

35. Hoyland JA, Le Maitre CL and Freemont AJ: Investigation of the role of IL-1 and TNF in matrix degradation in the intervertebral disc. Rheumatology (Oxford) 47: 809-814, 2008.

36. Korhonen T, Karppinen J, Malmivaara A, Autio R, Niinimäki J, Paimela L, Kyllönen E, Lindgren KA, Tervonen O, Seitsalo S and Hurri H: Efficacy of infliximab for disc herniation-induced sciatica: One-year follow-up. Spine (Phila Pa 1976) 29: 2115-2119, 2004.

37. Cohen SP, Bogduk N, Dragovich A, Buckenmaier CC III, Griffith S, Kurihara C, Raymond J, Richter PJ, Williams N and Yaksh TL: Randomized, double-blind, placebo-controlled, dose-response, and preclinical safety study of transforaminal epidural etanercept for the treatment of sciatica. Anesthesiology 110: 1116-1126, 2009.

38. Aluyen JK, Ton QN, Tran T, Yang AE, Gottlieb HB and Bellanger RA: Resveratrol: Potential as anticancer agent. J Diet Suppl 9: 45-56, 2012.

39. Wu JM, Hsieh TC and Wang Z: Cardioprotection by resveratrol: A review of effects/targets in cultured cells and animal tissues. Am J Cardiovasc Dis 1: 38-47, 2011

40. Recio MC, Andujar I and Rios JL: Anti-inflammatory agents from plants: Progress and potential. Curr Med Chem 19: 2088-2103, 2012.

41. Wuertz K, Quero L, Sekiguchi M, Klawitter M, Nerlich A, Konno S, Kikuchi S and Boos N: The red wine polyphenol resveratrol shows promising potential for the treatment of nucleus pulposus-mediated pain in vitro and in vivo. Spine (Phila $\mathrm{Pa}$ 1976) 36: E1373-E1384, 2011.

42. Li Y, Li K, Han X, Mao C, Zhang K, Zhao T and Zhao J: The imbalance between TIMP3 and matrix-degrading enzymes plays an important role in intervertebral disc degeneration. Biochem Biophys Res Commun 469: 507-514, 2016.

43. Roy S, Sannigrahi S, Majumdar S, Ghosh B and Sarkar B: Resveratrol regulates antioxidant status, inhibits cytokine expression and restricts apoptosis in carbon tetrachloride induced rat hepatic injury. Oxid Med Cell Longev 2011: 703676, 2011

44. Culpitt SV, Rogers DF, Fenwick PS, Shah P, De Matos C, Russell RE, Barnes PJ and Donnelly LE: Inhibition by red wine extract, resveratrol, of cytokine release by alveolar macrophages in COPD. Thorax 58: 942-946, 2003.

45. Kang OH, Jang HJ, Chae HS, Oh YC, Choi JG, Lee YS, Kim JH, Kim YC, Sohn DH, Park H and Kwon DY: Anti-inflammatory mechanisms of resveratrol in activated HMC-1 cells: Pivotal roles of NF-kappaB and MAPK. Pharmacol Res 59: 330-337, 2009.

46. Li X, Phillips FM, An HS, Ellman M, Thonar EJ, Wu W, Park D and Im HJ: The action of resveratrol, a phytoestrogen found in grapes, on the intervertebral disc. Spine (Phila Pa 1976) 33: 2586-2595, 2008

47. Kayama S, Konno S, Olmarker K, Yabuki S and Kikuchi S: Incision of the annulus fibrosus induces nerve root morphologic, vascular, and functional changes. An experimental study. Spine (Phila Pa 1976) 21: 2539-2543, 1996.

48. Ulrich JA, Liebenberg EC, Thuillier DU and Lotz JC: ISSLS prize winner: Repeated disc injury causes persistent inflammation. Spine (Phila Pa 1976) 32: 2812-2819, 2007.

49. Shamji MF, Allen KD, So S, Jing L, Adams SB Jr, Schuh R, Huebner J, Kraus VB, Friedman AH, Setton LA and Richardson WJ: Gait abnormalities and inflammatory cytokines in an autologous nucleus pulposus model of radiculopathy. Spine (Phila Pa 1976) 34: 648-654, 2009. 